\title{
Claude Lefèvre
}

\section{PHILIPPE LALOYAUX}

\section{Note sur les problèmes d'ordonnancement PERT aléatoires}

Revue française d'automatique, d'informatique et de recherche opérationnelle. Recherche opérationnelle, tome 19, n 1 (1985), p. 27-33.

<http://www.numdam.org/item?id=RO_1985_19_1_27_0>

(C) AFCET, 1985, tous droits réservés.

L'accès aux archives de la revue « Revue française d'automatique, d'informatique et de recherche opérationnelle. Recherche opérationnelle » implique l'accord avec les conditions générales d'utilisation (http://www.numdam.org/ legal.php). Toute utilisation commerciale ou impression systématique est constitutive d'une infraction pénale. Toute copie ou impression de ce fichier doit contenir la présente mention de copyright.

\section{Numdam}

Article numérisé dans le cadre du programme

Numérisation de documents anciens mathématiques

http://www.numdam.org/ 
R.A.I.R.O. Recherche opérationnelle/Operations Research

(vol. 19, n 1 , février 1985, p. 27 à 33 )

\title{
NOTE SUR LES PROBLĖMES D'ORDONNANCEMENT PERT ALÉATOIRES ( $*$ )
}

\author{
par Claude Lefèvre $\left({ }^{1}\right)$ et Philippe Laloyaux $\left({ }^{2}\right)$
}

\begin{abstract}
Résumé. - Le but de cette note est double. Nous présentons d'abord un bref résumé des différentes approches développées dans la littérature pour étudier les problèmes d'ordonnancement PERT aléatoires. Ceci nous permet, en particulier, de situer clairement la méthode approchée récente de Melin parmi les autres méthodes proposées. Nous construisons ensuite des bornes pour la durée moyenne d'exécution du projet. Ces bornes, faciles à calculer, dépendent uniquement des moyennes et des variances des durées des tâches qui composent le projet.
\end{abstract}

Mots clés : Ordonnancement; Maximum de variables aléatoires; Bornes.

Abstract. - The purpose of this note is twofold. We first present a brief survey of the different approaches developed in the literature to study the probabilistic PERT scheduling problems. This allows us, in particular, to situate clearly the recent approximative method of Melin among the other methods proposed. We then construct bounds for the expected total duration of the project. These bounds can be easily computed and depend only on the means and the variances of the durations of the activities composing the project.

Keywords: Scheduling; Maximum of random variables; Bounds.

\section{LE PROBLÈME POSÉ}

Un projet d'ordonnancement a comme objet d'aider le décideur à planifier et contrôler l'exécution d'un projet décomposable en un nombre fini $n$ de tâches $i, 1 \leqq i \leqq n$. Il est dit élémentaire lorsque les tâches $i$, de durées d'exécution $\mathrm{d}_{\mathrm{i}}$, sont soumises uniquement à des contraintes de postériorité stricte et que le critère retenu est la minimisation de la durée totale d'exécution du projet.

La méthode PERT, développée initialement par Malcolm, Roseboom, Clark et Fazar [8], est sans doute la méthode la plus connue qui permette

(*) Reçu en novembre 1983 .

$\left({ }^{1}\right)$ Université Libre de Bruxelles, Institut de Statistique, C.P. 210, boulevard du Triomphe, B-1050 Bruxelles.

$\left({ }^{2}\right)$ Compagnie d'Assurance EUR AVIE, 152, avenue de Malakoff, 750016 Paris.

R.A.I.R.O. Recherche opérationnelle/Operations Research, 0399-0559/85/01 2707/\$2.70

(C) AFCET-Gauthier-Villars. 
d'étudier un tel problème. Elle repose sur la construction d'un graphe sans circuit dont les arcs et leurs longueurs représentent les tâches et leurs durées. La durée minimale de réalisation du projet correspond à la longueur du plus long chemin joignant le sommet initial $x_{0}$ " début du travail » au sommet terminal $x_{t}$ " fin du travail ».

Le cas généralement traité dans la littérature est celui où les durées $d_{i}$ sont des constantes. Le chemin le plus long peut alors être aisément déterminé au moyen d'un algorithme; voir, par exemple, Roy [13]. Il est clair cependant que dans la réalité, ces durées sont, le plus souvent, non pas des constantes mais bien des variables aléatoires. La durée minimale d'exécution du projet, notée $X$, est alors une variable aléatoire qui peut se définir par :

$$
X=\max _{1 \leqq j \leqq J} L_{j}
$$

où les variables aléatoires $L_{j}$ représentent les longueurs des chemins, supposés en nombre $J$, joignant les sommets $x_{0}$ et $x_{t}$. Ces chemins peuvent être déterminés au moyen d'un algorithme classique; voir, par exemple, Roy [13]. Sous l'hypothèse, fréquemment admise, que les durées $d_{i}$ sont des variables aléatoires indépendantes, la distribution de la longueur $L_{j}$ du chemin $j$, $1 \leqq j \leqq J$, est la convoluée des distributions des durées des tâches qui le composent. Les variables $L_{j}$ ne sont cependant indépendantes que dans le cas très particulier où tous les chemins sont parallèles. L'étude de la durée minimale d'exécution du projet revient donc à celle du maximum d'un ensemble fini de variables aléatoires le plus souvent corrélées et non equidistribuées. Il s'agit là d'un problème très difficile à traiter.

De nombreuses méthodes, exactes ou approchées, ont été développées pour calculer la distribution de $X$ et notamment sa moyenne $E(X)$. Dans un article récent paru dans ce Journal, Melin [10] a proposé une nouvelle solution approchée pour ce calcul. On peut cependant regretter qu'aucune des autres méthodes ne soit mentionnée dans cet article. Dans la section 2, nous présentons un bref résumé des principaux travaux parus actuellement sur ce problème. Ceci nous permet, en particulier, de situer clairement l'approche de Melin parmi les autres approches envisagées.

L'application de ces méthodes suppose au préalable que les distributions des durées des tâches sont connues exactement. En pratique, il est parfois très difficile de disposer de cette information. Dans un tel cas, néanmoins, le. décideur pourra généralement connaître les durées minimale, maximale et modale de chacune des tâches. Nous indiquons dans la section 3 comment construire très facilement, à partir de ces seules données, des bornes pour la durée moyenne d'exécution du projet.

R.A.I.R.O. Recherche opérationnelle/Operations Research 


\section{UN BREF SURVOL DES PRINCIPALES METHODES D'ANALYSE}

Les différentes approches pour déterminer la distribution de la variable $X$ supposent le plus souvent que les durées d'exécution des tâches $d_{i}$ sont des variables aléatoires indépendantes. Elles peuvent être classées essentiellement en cinq types.

La première approche, développée initialement par Malcolm, Roseboom, Clark et Fazar [8], consiste à résoudre d'abord le problème PERT déterministe obtenu en remplaçant chacune des variables $d_{i}$ par sa moyenne $E\left(d_{i}\right)$ calculée à partir d'une distribution bêta. Le chemin critique ainsi déterminé est ensuite supposé contenir un nombre suffisamment important de tâches que pour pouvoir approcher la distribution de $X$ par une distribution normale.

La seconde approche est la méthode analytique de calcul de la distribution exacte de $X$. Son principe est présenté et illustré dans le livre d'Elmaghraby [3]. Martin [9] a complété cette méthode en développant un algorithme de calcul très efficient dans le cas où les $d_{i}$ ont une fonction de densité polynomiale.

La troisième approche consiste à procéder comme si les longueurs $L_{j}$ des différents chemins de $x_{0}$ à $x_{t}$ étaient des variables aléatoires indépendantes. Cette méthode a été appliquée par Sculli [14] dans le cas où les $d_{i}$ ont une distribution normale et par Melin [10] dans le cas où les $d_{i}$ ont une distribution bêta d'un type particulier.

La quatrième approche est la méthode de simulation Monte Carlo. Van Slyke [16] a décrit comment simuler de manière directe les problèmes PERT aléatoires et divers auteurs (Hartley et Wortham [5] et Garman [6], par exemple) ont montré comment améliorer les résultats en recourant en plus à des méthodes analytiques.

La cinquième approche, plus récente, consiste à borner la fonction de répartition de $X$. Robillard et Trahan [12] ont calculé une borne supérieure pour cette fonction tandis que Shogan [15] a déterminé des fonctions de répartition qui la bornent supérieurement et inférieurement.

Un grand nombre de travaux ont été également développés pour borner la durée moyenne $E(X)$. Beaucoup d'entre eux fournissent des bornes inférieures; citons notamment celles construites par Fulkerson [4], Elmaghraby [2] et Robillard et Trahan [11]. Sur base des bornes dérivées pour la fonction de répartition de $X$, Robillard et Trahan [12] et Shogan [15] ont pu calculer des bornes supérieure et inférieure pour $E(X)$. 
Ceci complète un très bref survol des principales approches. Elles font toutes l'hypothèse préalable que les distributions des $d_{i}$ sont connues avec exactitude, et leur mise en application requiert souvent un traitement numérique assez important.

\section{BORNES POUR LA DURÉE MOYENNE D'EXECUTION DU PROJET}

Les distributions des durées des tâches sont parfois difficiles à estimer. Par contre, pour chacune des tâches $i$, le décideur peut généralement connaître la durée minimale, ou "optimiste ", $a_{i}$, la durée maximale, ou " pessimiste", $b_{i}$, et la durée "la plus probable ", $M_{i}$. Sur base de ces seules données, il est impossible d'étudier le problème PERT aléatoire par l'une des méthodes rappelées à la section 2. Quelques renseignements utiles sur la durée du projet peuvent cependant être obtenus très facilement en procédant comme suit.

Nous faisons l'hypothèse habituelle que les durées $d_{i}$ sont des variables aléatoires indépendantes. De plus, nous supposons que pour chacune des tâches $i$, il est possible d'évaluer, à partir de $a_{i}, b_{i}$ et $M_{i}$, la durée moyenne $m_{i} \equiv E\left(d_{i}\right)$ et la variance $\sigma_{i}^{2} \equiv \operatorname{Var}\left(d_{i}\right)$. Il s'agit là de la seule hypothèse de travail supplémentaire. Pour calculer une valeur approchée de ces paramètres, on peut suivre une des différentes méthodes proposées habituellement dans la littérature. Ainsi par exemple, on peut utiliser les formules "classiques " de Malcolm, Roseboom, Clark et Fazar [8], ou encore les formules plus " réalistes » de MacCrimon et Ryavec [7] :

$$
\left\{\begin{array}{c}
m_{i}=\left(a_{i}+M_{i}+b_{i}\right) / 3 \\
\sigma_{i}^{2}=\left[\left(b_{i}-a_{i}\right)^{2}+\left(M_{i}-a_{i}\right)\left(M_{i}-b_{i}\right)\right] / 18
\end{array}\right.
$$

obtenues dans le cas où les $d_{i}$ admettent une distribution triangulaire.

Un premier renseignement qui peut être obtenu aisément est le domaine de variations de $X$. En effet, les durées "optimiste ", $a$, et " pessimiste ", $b$, d'exécution du projet se calculent simplement en résolvant les problèmes PERT déterministes obtenus en remplaçant chaque variable $d_{i}$ par ses durées minimale $a_{i}$ et maximale $b_{i}$, respectivement.

Dans la plupart des cas, cependant, ce que demandera avant tout le décideur, c'est de connaître, ne fut-ce qu'approximativement, la durée moyenne $E(X)$. Nous allons montrer ci-dessous comment obtenir des bornes pour $E(X)$. A partir de la définition (1) de $X$, nous déduisons directement que :

$$
E(X) \geqq \underline{E}(X) \equiv \max _{1 \leqq j \leqq J} E\left(L_{j}\right) .
$$

R.A.I.R.O. Recherche opérationnelle/Operations Research 
Pour calculer $\underline{E}(X)$, il suffit alors de calculer la longueur du plus long chemin dans le modèle PERT déterministe obtenu en remplaçant chaque variable $d_{i}$ par sa moyenne $E\left(d_{i}\right)$. D'autre part, en utilisant une propriété sur les statistiques d'ordre établie par Arnold et Groeneveld [1], on peut montrer que :

$$
E(X) \leqq \bar{E}(X) \equiv \mu+\sqrt{[(J-1) / J] \sum_{j=1}^{J}\left\{\operatorname{Var}\left(L_{j}\right)+\left[E\left(L_{j}\right)-\mu\right]^{2}\right\}},
$$

où :

$$
\mu \equiv(1 / J) \sum_{j=1}^{J} E\left(L_{j}\right) .
$$

Il est clair que pour chaque chemin $j, E\left(L_{j}\right)$ et $\operatorname{Var}\left(L_{j}\right)$ sont respectivement égales à la somme des moyennes et des variances des durées des tâches qui composent ce chemin; ces paramètres peuvent donc être déterminés immédiatement à partir des données $m_{i}$ et $\sigma_{i}^{2}$. Signalons que la borne inférieure (3) est bien connue tandis que la borne supérieure (4) n'a jamais été utilisée, à notre connaissance, dans l'étude des problèmes d'ordonnancement.

A titre d'illustration, considérons d'abord le graphe suivant :

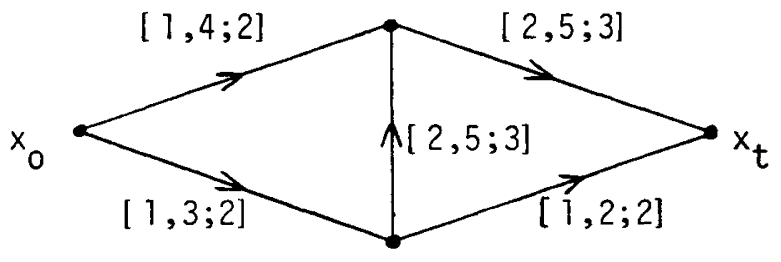

Figure 1.

où les nombres associés à chacun des arcs $i$ représentent respectivement $\left[a_{i}, b_{i} ; M_{i}\right]$. Les moyennes et variances $m_{i}$ et $\sigma_{i}^{2}$ sont estimées à partir des formules (2). Nous obtenons alors :

$$
[a, b]=[5,13] ; \quad \underline{E}(X)=8,67 ; \quad \bar{E}(X)=9,12 .
$$

Reprenons maintenant le graphe suivant envisagé dans [12] :

$$
\text { voir la figure } 2
$$

où pour chaque arc $i$, la durée $d_{i}$ correspondante admet une distribution uniforme sur l'intervalle $\left[a_{i}, b_{i}\right]$ indiqué. Les $m_{i}$ et $\sigma_{i}^{2}$ que nous utilisons ici sont les vraies moyennes et variances des $d_{i}$. Nous déduisons alors :

$$
[a, b]=[15,26] ; \quad \underline{E}(X)=20,50 ; \quad \bar{E}(X)=21,51 .
$$




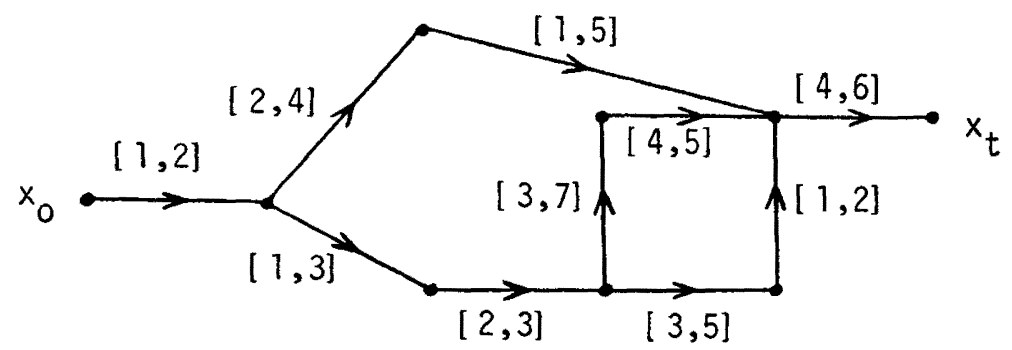

Figure 2.

La borne inférieure est celle obtenue dans [12]. La borne supérieure est un peu moins bonne (dans [12], $\bar{E}(X)=20,56$ ), mais elle est beaucoup plus facile à calculer, et plus générale et plus fiable puisqu'elle dépend uniquement des moyennes et variances des distributions des $d_{i}$.

\section{BIBLIOGRAPHIE}

1. B. C. Arnold et R. A: Groeneveld, Bounds on Expectations of Linear Systematic Statistics Based on Dependent Samples, The Annals of Statistics, vol. 7, $\mathrm{n}^{\circ} 1$, 1979 , p. $220-223$.

2. S. E. Elmaghraby, On the Expected Duration of PERT Type Networks, Management Science, vol. 13, ${ }^{\circ} 5,1967$, p. 229-306.

3. S. E. Elmaghraby, Activity Networks, John Wiley and Sons, Inc., New York, 1977.

4. D. R. Fulkerson, Expected Critical Path Lengths in PERT Networks, Operations Research, vol. $10, \mathrm{n}^{\circ} 6,1962$, p. 808-817.

5. H. O. Hartley et A. W. Wortham, A Statistical Theory for PERT Critical Path Analysis, Management Science, vol. 12, $\mathrm{n}^{\circ} 10,1966$, p. 469-481.

6. M. B. Garman, More on Conditional Sampling in the Simulation of Stochastic Networks, Management Science, vol. 19, $\mathrm{n}^{\circ}$ 1, 1972, p. 90-95.

7. K. R. Maccrimmon et C. A. Ryavec, An Analytical Study of the PERT Assumptions, Operations Research, vol. 12, $\mathrm{n}^{\circ} 1,1964$, p. 16-37.

8. P. G. Malcolm, J. H. Roseboom, C. E. Clark et W. Fazar, Application of a Technique for Research and Development, Operations Research, vol. 7, $\mathrm{n}^{\circ}$ 5, 1959, p. 646-669.

9. J. J. Martin, Distribution of Time Through a Directed, Acyclic Network, Operations Research, vol. 13, $\mathrm{n}^{\circ} 1,1965$, p. 46-66.

10. J. P. Melin, Proposition d'une Solution Approchée pour l'Étude du Maximum de Plusieurs Variables Aléatoires, R.A.I.R.O.-Rech. Op., vol. 17, n $^{\circ}$ 2, 1983, p. $175-$ 191.

11. P. Robillard et M. Trahan, Expected Completion Time in PERT Networks, Operations Research, vol. 24, $\mathrm{n}^{\circ} 1,1976$, p. 177-182.

R.A.I.R.O. Recherche opérationnelle/Operations Research 
12. P. Robillard et M. Trahan, The Completion Time of PERT Networks, Operations Research, vol. $25, \mathrm{n}^{\circ} 1,1977$, p. 15-29.

13. B. Roy, Algèbre Moderne et Théorie des Graphes, Dunod, Paris, 1970.

14. D. Scully, The Completion Time of PERT Networks, J. Operational Research Society, vol. $34, \mathrm{n}^{\circ} 2,1983$, p. 155-158.

15. A. W. Shogan, Bounding Distributions for a Stochastic PERT Network, Networks, vol. $7, \mathrm{n}^{\circ} 4,1977$, p. $359-381$.

16. R. M. VAN SLYKe, Monte Carlo Methods and the PERT Problem, Operations Research, vol. 11, n ${ }^{\circ} 5,1963$, p. 839-860. 